\title{
ANÁLISIS DE PAUSAS Y EJECUCIONES PARA EL ESTUDIO DE PROCESOS DE ESCRITURA
}

\author{
Paula López \\ plopg@unileon.es \\ Raquel Fidalgo \\ Departamento de Psicología, Sociología y Filosofía, Universidad de León, España
}

Fecha de Recepción: 14 Febrero 2018

Fecha de Admisión: 10 Abril 2018

\section{RESUMEN}

Uno de los métodos online de mayor trascendencia en los últimos años para la evaluación del proceso de escritura es el estudio de la dinámica temporal de escritura a través del análisis de las pausas y ejecuciones. Además de ser uno de los métodos de naturaleza online menos intrusivos, proporciona información de gran valor sobre el proceso de escritura. A pesar de ello, existe una falta de consistencia respecto al tipo de medidas que pueden utilizarse y la información que se puede obtener a partir de su análisis. Por ello, a partir de la realización de una revisión exhaustiva de estudios, el presente trabajo pretende proporcionar una visión detallada del tipo de medidas relacionadas con el análisis de pausas y ejecuciones así como el tipo de información que se puede inferir a través de las mismas. Igualmente, se aporta información sobre las herramientas necesarias para la recogida y tratamiento de datos. Por último, se discutirán las mayores aportaciones derivadas de la implementación de dicha metodología así como sus limitaciones y dificultades. A partir de esta revisión, se pretende aportar información valiosa que sea útil para el diseño de nuevos estudios de investigación en los que se consideren este tipo de medidas. Estudio financiado a través del proyecto EDU2015-67484-P, MINECO/FEDER concedido a la Dra. Fidalgo y una beca predoctoral (FPU 13/06428) concedida a P. López.

Palabras clave: proceso de escritura; evaluación on-line; análisis de pausas y ejecuciones; evaluación procesos cognitivos

\section{ABSTRACT \\ Analysis of pauses and bursts for the study of writing processes. \\ One of the most relevant online methods for the assessment of the writing process in the last few years has been the study of the temporal dynamics of writing through the analysis of pauses and executions. In addition to being one of the less intrusive online methods, it provides valuable information about writing processes. Despite this, there is a certain lack of consistency regarding}




\section{ANÁLISIS DE PAUSAS Y EJECUCIONES PARA EL ESTUDIO DE PROCESOS DE ESCRITURA}

the kind of measures that can be considered and the information that can be obtained from their analysis. Therefore, based on an exhaustive revision of studies, this work aims to provide a detailed view of the kind of measures related to the analysis of pauses and bursts as well as the kind of information that can be inferred from them. Information is also provided on the tools required for data collection and processing. Finally, the major contributions derived from the implementation of this methodology will be discussed, as well as the limitations and difficulties that should be considered for its implementation. From this review, it is intended to provide valuable information that will be useful for the conceptualization and design of new writing research studies in which this type of measures are considered. Research funded through the project EDU2015-67484-P, MINECO/FEDER awarded to Dra. Fidalgo and a predoctoral fellowship (FPU 13/06428) granted to P. López.

Keywords: writing process; on-line assessment; analysis of pauses and bursts; cognitive processes assessment

\section{INTRODUCCIÓN}

En los años 80 se da un cambio de concepción en el estudio de la escritura a nivel científico (Levy \& Olive 2002). Dicho cambio viene marcado por la imposibilidad de conocer qué pasa a nivel cognitivo durante la escritura a través del análisis del producto textual. Como consecuencia, empieza a surgir un interés por el estudio del proceso de escritura, dando lugar a la aparición de nuevos métodos y técnicas de investigación necesarios para su evaluación. Una de las primeras técnicas utilizadas para la evaluación del proceso de escritura fue el análisis de protocolos de pensamiento en voz alta. Concretamente, éste fue el método que permitió desarrollar el primer modelo cognitivo de escritura (Hayes \& Flower, 1980). Aún a día de hoy, este es uno de los modelos más importantes dado que fue el primero en identificar los procesos cognitivos activados durante la escritura como son la planificación, redacción y revisión textual y determinar el carácter recursivo con el que dichos procesos son empleados durante la composición. La aparición de este estudio marcó un punto de inflexión respecto al tipo de investigaciones llevadas a cabo en el ámbito de escritura, centradas desde entonces en el estudio de los procesos implicados en la escritura o la influencia de la memoria operativa entre otros, a través del uso de novedosos instrumentos para su evaluación.

En función del momento de aplicación, los instrumentos de evaluación pueden ser clasificados como herramientas off-line u on-line (Van der Pool, 1996). Los instrumentos off-line son aquellos en los que los datos se recogen de manera independiente a la tarea de escritura, tales como, la realización de cuestionarios o entrevistas. En la actualidad, existe una gran controversia respecto al uso de este tipo de instrumentos para la evaluación del proceso de escritura dado que varios estudios han evidenciado que la información obtenida a través de los mismos es incompleta y generalmente no se corresponde con la realidad (Cromley \& Azevedo, 2006; Tillema, Van den Bergh, Rijlaarsdam, \& Sanders, 2011). Por su parte, los instrumentos de evaluación on-line son aquellos en los que los datos sobre el proceso de escritura son recogidos en tiempo real, es decir, durante la realización de la propia tarea. Esto hace que sean las herramientas idóneas para el estudio del proceso de escritura (Tillema et al., 2011). En las últimas décadas hemos sido testigos de un creciente interés por el uso de este tipo de instrumentos con dos objetivos principales, conocer cuándo y cómo se implementan los procesos cognitivos durante la composición así como explorar la influencia que ejercen diferentes factores relacionados con la situación o con las características del escritor sobre el proceso de escritura (Olive, 2010).

En general, las técnicas on-line dentro del ámbito de estudio de la escritura se centran en la valoración de sus características funcionales a través de técnicas de pensamiento en voz alta, el análisis de la demanda cognitiva con diseños de doble o triple tarea o el análisis de la dinámica tempo- 
ral a través del estudio de pausas y ejecuciones (Olive, 2010). El presente trabajo se centra en el análisis de las pausas y ejecuciones por ser el método menos intrusivo y uno de los que mayores posibilidades ofrece de los anteriormente mencionados.

Las pausas y ejecuciones son las dos actividades básicas directamente observables que tienen lugar durante la escritura de manera natural. Kaufer, Hayes y Flower (1986) fueron los primeros en identificar que los escritores adultos componían sus textos en periodos de ejecución de aproximadamente nueve palabras separadas por pausas con una duración mayor a dos segundos. A partir del análisis de pausas y ejecuciones es posible inferir la activación de ciertas operaciones cognitivas midiendo el tiempo de procesamiento durante la producción textual a partir del registro de datos espaciales y temporales (Barbier \& Spinelli-Jullien, 2009).

A pesar de ser uno de los instrumentos de evaluación qué más posibilidades y ventajas ofrece para el estudio del proceso de escritura, existe una falta de consistencia respecto al tipo de medidas consideradas para su estudio así como la información que puede ser obtenida a partir de su análisis. Por ello, el objetivo del presente trabajo se centra en proporcionar una visión detallada de este tipo de medidas, la información que puede extraerse a partir de su análisis y las herramientas necesarias para la recogida y tratamiento de datos. Igualmente, se discutirá el valor de este tipo de medidas así como sus limitaciones.

\section{ANÁLISIS DE PAUSAS EN COMPOSICIÓN ESCRITA}

Las pausas se definen como aquellos periodos en los que se interrumpe la escritura (Olive, 2010). Varias investigaciones han demostrado que los escritores pasan la mitad del tiempo de composición sin escribir (Alves, Castro, Sousa, \& Strömqvist, 2007). En base a la revisión realizada, dos tipos de medidas son consideradas para el estudio de las pausas como son la duración y su localización en el texto.

Un primer aspecto de gran importancia para el análisis de pausas es determinar el umbral de tiempo o duración mínima considerada para el análisis de las mismas. Existe una clara falta de consistencia respecto a la duración a considerar para el establecimiento de pausas, siendo un aspecto crítico para la comparación de estudios (Medimorec \& Risko, 2017). A partir de la revisión realizada, se ha podido comprobar que los umbrales temporales varían notablemente en función del objetivo de la investigación, considerándose desde los 15 milisegundos hasta más de 10 segundos.

Generalmente, aquellas investigaciones interesadas en el estudio de procesos relacionados con la transcripción consideran pausas entre los 15 milisegundos y los 2 segundos (ver Alamargot, Plane, Lambert, \& Chesnet, 2010; Limpo \& Alves, 2017; Puntry, Barnett, Wilmut, y Plumb, 2013; 2014; Wengelin, 2006). Estos estudios han mostrado, que las habilidades de transcripción, cuando no están automatizadas, suponen una alta demanda cognitiva provocando que el escritor tenga que parar para atender dicha demanda. Así, Limpo y Alves (2017) mostraron que una instrucción centrada en la mejora de habilidades de transcripción en alumnos de $2^{\circ}$ de primaria, redujo el número de las pausas menores a dos segundos relacionadas con la transcripción.

Por su parte, las pausas mayores de 2 segundos han sido empleadas y relacionadas con la activación de procesos de alto nivel cognitivo como la planificación o la revisión (Alves et al., 2007; Chenoweth \& Hayes, 2001; Kaufer et al., 1986; Van Waes \& Schellens, 2003; Wengelin, 2007). Por ejemplo, Van Waes \& Schellens (2003), establecieron que más de la mitad de las pausas mayores a 3 segundos iban seguidas por revisiones.

Por otra parte, varios estudios han puesto de manifiesto que la duración de las pausas parece variar en función de su localización en el texto (ver Foulin, 1998; Spelman Miller, 2000; Van Hell, Verhoeven, \& Van Beijsterveldt, 2008; Van Waes \& Schellens, 2003; Wengelin, 2006). Estos estu- 


\section{ANÁLISIS DE PAUSAS Y EJECUCIONES PARA EL ESTUDIO DE PROCESOS DE ESCRITURA}

dios evidenciaron que las pausas son más largas a medida que aumenta el nivel sintáctico de la unidad con la que se asocia, tanto en escritores jóvenes como adultos. Así por ejemplo, las pausas antes de un párrafo son más largas que aquellas realizadas antes de una oración. Esto sugiere que tanto los escritores principiantes como los más hábiles necesitan más tiempo para planificar cuanto más larga es la unidad sintáctica que van a escribir, aumentando así la demanda cognitiva de esta actividad.

Por último, este tipo de medidas han sido utilizadas para explorar aspectos relacionados con las dificultades de escritura mostradas en poblaciones específicas como sujetos con dislexia. Por ejemplo, Wengelin (2007), observó que los adultos con dislexia paraban dos veces más que los escritores sin dislexia antes de las palabras y 10 veces más durante la escritura de las mismas en comparación con sujetos sin dislexia. Similares resultados han sido encontrados en alumnos con dificultades de decodificación o alumnado con trastornos en el desarrollo de la coordinación en comparación con alumnos de desarrollo típico (Puntry, et al., 2013; 2014; Torrance, Rønneberg, Johansson \& Uppstad, 2016). Estos resultados sugieren que la lentitud que caracteriza la escritura de los sujetos en estas poblaciones se debe a que éstos centran su atención en aspectos relacionados con la transcripción, siendo un proceso de gran coste cognitivo que consume gran parte de los recursos de la memoria operativa.

\section{ANÁLISIS DE EJECUCIONES EN COMPOSICIÓN ESCRITA}

Las ejecuciones pueden ser definidas como el tiempo comprendido entre dos pausas consecutivas durante las cuales tienen lugar diferentes procesos de escritura (Olive, 2010). El interés de los investigadores por los periodos de ejecución se centra en la consideración de su longitud, entendida como número de palabras escritas o duración en el tiempo.

En primer lugar la longitud de dichos periodos, parece estar relacionada con la habilidad de escritura. Parece ser que el tipo de procesos que se activan durante las ejecuciones depende de la automatización de los procesos de bajo nivel relacionados con la transcripción. Alves y colaboradores (2007), encontraron que los universitarios con buenas habilidades de transcripción escribían ejecuciones más largas que aquellos con menor habilidad, dándose una correlación positiva entre la mayor longitud de las ejecuciones y la calidad textual final. Igualmente, Alves (2013) demostró que dificultar la transcripción pidiendo a escritores adultos que escribiesen en letras mayúsculas o en un teclado cifrado, reducía significativamente la longitud de las ejecuciones. A pesar de que en estos estudios se trabajó con escritores adultos, en niños también parece darse esta relación. Alves, Branco, Castro y Olive (2012) y Conelly, Drockel, Walter y Critten (2012), observaron que los alumnos con mejores habilidades de escritura a mano escribían ejecuciones más largas que aquellos con menor habilidad. Adicionalmente, Conelly et al., (2012) encontraron que los alumnos de desarrollo típico producían ejecuciones más largas que aquellos con alguna dificultad y que las ejecuciones más largas se relacionan de forma positiva con la fluidez y la calidad textual. Por su parte, Alves y Limpo (2015), encontraron que la longitud de las ejecuciones incrementa durante la escolarización a medida que los procesos de bajo nivel cognitivo se automatizan, disminuyendo así la demanda cognitiva de dichos procesos.

Por su parte, la capacidad de transformar las ideas en palabras escritas también parece influir en la longitud de las ejecuciones. En un estudio pionero, Kaufer et al., (1986) encontraron que los escritores profesionales escribían más palabras por ejecución que los estudiantes universitarios. Estos autores concluyeron que las diferencias encontradas se debían a que los escritores expertos tenían más facilidad para expresar sus ideas que los escritores universitarios. En esta misma línea, Chenoweth y Hayes (2001) encontraron que las composiciones de alumnos universitarios contení- 
an ejecuciones más largas cuando éstos escribían en su lengua materna en comparación con la escritura en una segunda lengua.

Por último, varias investigaciones han explorado qué procesos son activados durante los periodos de ejecución. Alves, Castro y Olive (2008) y Olive, Alves y Castro (2009) investigaron qué tipo de actividades cognitivas tenían lugar durante los periodos de ejecución en estudiantes universitarios. Estos autores mostraron que la formulación de ideas es la operación que más se activa durante las ejecuciones. Dichos resultados sugieren que la formulación de ideas es una de las actividades con menor coste cognitivo en comparación con procesos de planificación o revisión, lo que permite que dicho proceso pueda ser activado de forma concomitante a la escritura en escritores expertos. A pesar de ello, y en contra de las hipótesis de los investigadores, los resultados confirmaron que los procesos de planificación y revisión también tenían lugar durante los periodos de ejecución, aunque en un porcentaje significativamente menor. Según los autores, podría ser que dichos procesos complejos fueran activados al inicio de la ejecución y a medida que los escritores se involucran en ellos y aumenta la demanda cognitiva, se produce la interrupción de la ejecución. Por su parte, Olive y Kellog (2002), observaron que los escritores jóvenes eran incapaces de activar procesos de alto nivel durante los periodos de ejecución, para lo que tenían que suspender la escritura y pensar sobre sus textos.

\section{HERRAMIENTAS NECESARIAS PARA LA RECOGIDA Y TRATAMIENTO DE DATOS}

Las herramientas para la recogida de datos de pausas y ejecuciones han evolucionado en los últimos años notablemente. Los tediosos sistemas utilizados en el pasado han sido sustituidos en la actualidad por grabaciones digitales realizadas a través de diversos soportes como tabletas digitales, ordenadores o bolígrafos digitales. Todos estos instrumentos son herramientas no intrusivas que permiten recoger los datos de manera precisa y fiable. Sin embargo, en la escritura a través del ordenador se debe considerar el nivel de mecanografía de los escritores, ya que esto puede influir en el proceso de escritura (Barbier \& Spinelli-Jullien, 2009). Este aspecto sería especialmente relevante cuando se trabaja con escritores jóvenes. Por ello, en la actualidad existe una preferencia por el uso de aquellos métodos como la tableta gráfica o los bolígrafos digitales que evalúan el proceso escritor a partir de la escritura a mano.

Del mismo modo, independientemente del soporte utilizado, los datos recogidos deben ser tratados por un software específicamente diseñado para ello. Los programas Scriptlog e Imputlog son los softwares que mejores posibilidades ofrecen para la recogida de datos a través del ordenador (Van Waes, Leitjen, Wengelin, \& Lindgren, 2012). A partir de su utilización se recoge información sobre las pulsaciones, movimientos de ratón, acciones de revisión, etc., así como su distribución temporal. Por su parte, el programa Eye \& Pen es el software más utilizado para la recogida de datos a través del uso de tabletas digitales. Una de sus mayores ventajas es que recoge los datos a través de los movimientos del lápiz asociándolos con movimientos oculares (Alamargot et al., 2010). Igualmente, el HandSpy es un software específicamente diseñado para la recogida de datos a través de la utilización de bolígrafos digitales. A partir de su uso, se obtiene información sobre la duración de las pausas y su localización, la longitud de las ejecuciones o tiempo de procesamiento entre otros (Limpo \& Alves, 2015; 2017). Finalmente, en los últimos años ha aparecido un software denominado MarkWrite, el cual proporciona la misma información que los softwares anteriores en un entorno digital más cómodo y rápido para la extracción de datos. La mayor ventaja de este software, es que permite trabajar con datos provenientes de diversos soportes como las tabletas o los bolígrafos digitales.

Por todo ello, en función de los objetivos de la investigación y el soporte digital utilizado es posi- 


\section{ANÁLISIS DE PAUSAS Y EJECUCIONES PARA EL ESTUDIO DE PROCESOS DE ESCRITURA}

ble utilizar una serie de softwares que sin dura facilitarán el tratamiento de datos y permitirán extraer información valiosa sobre el proceso de escritura.

\section{DISCUSIÓN Y CONCLUSIONES}

La revisión realizada permite identificar el análisis de pausas y ejecuciones como uno de los métodos idóneos para la evaluación de los procesos involucrados en la escritura. Una de las principales ventajas de éste método es que la recogida de datos se lleva a cabo de manera no intrusiva, sin interrumpir la tarea o aumentar la carga cognitiva de la misma, aspectos que pueden interferir y modificar el proceso de escritura. Igualmente, a partir de la revisión realizada se ha podido comprobar que las pausas y ejecuciones son indicadores de la habilidad escritora, aportando información sobre los diferentes procesos involucrados en la composición. Por todo ello, el análisis de pausas y ejecuciones es una de las opciones más pertinentes para el análisis del proceso de escritura.

Sin embargo, esta metodología también cuenta con una serie de limitaciones. En primer lugar, se debe considerar la dificultad en la interpretación de los datos, especialmente de las pausas. Según Schilperoord (2002), las pausas pueden ser debidas a factores cognitivos (ej. sobrecarga cognitiva), socio-psicológicos (ej. aprensión hacia la escritura) o razones físicas (ej. fatiga) entre otros. Con el fin de obtener información válida y fiable, un aspecto clave sería la identificación del suceso que provoca la pausa. Por otra parte, se ha visto que la activación de procesos cognitivos no tiene por qué darse de manera secuencial, sino que puede darse en paralelo (Olive \& Kellog, 2002). Por tanto, más atención debe prestarse a este aspecto con el fin de determinar cuántas operaciones cognitivas pueden llevarse a cabo durante un mismo periodo, especialmente en escritores expertos. Por último, como señalamos anteriormente, la falta de consistencia en el establecimiento del umbral temporal para el análisis de las pausas supone una limitación, ya que no permite la comparación de estudios. Este aspecto, es fundamental ya que determina tanto el análisis de las pausas, estableciendo aquellas de interés, así como de la longitud de las ejecuciones. Por tanto, futuras investigaciones deben desarrollarse para aportar más información sobre dichos aspectos, lo que sin duda incrementaría las ventajas y posibilidades de dicha metodología de evaluación.

Por último, este tipo de medidas también podrían ser implementadas con el fin de evaluar los efectos de la instrucción en composición escrita. Generalmente, los efectos de la instrucción son evaluados de manera general (ej. calidad textual) más que a través de medidas específicas del proceso (para excepción ver Alves et al., 2016; Limpo \& Alves, 2017). A pesar de la importancia de la evaluación general de la intervención, el análisis de medidas específicas del proceso es fundamental para aumentar el conocimiento sobre el funcionamiento las intervenciones eficaces y cómo pueden adaptarse a las necesidades de escritura de los estudiantes.

\section{REFERENCIAS BIBLIOGRÁFICAS}

Alamargot, D., Plane, S., Lambert, E., \& Chesnet, D. (2010). Using eye and pen movements to trace the development of writing expertise: Case studies of a 7th, 9th and 12th grader, graduate student, and professional writer. Reading and Writing, 23, 853-8 88. doi:10.1007/s11145-0099191-9

Alves, R. A. (2013). A mente enquanto escreve: A automatização da execução motora na composição escrita [The mind while writing: The automatization of motor execution in written composition]. Lisbon, Portugal: Fundação Calouste Gulbenkian.

Alves, R. A., \& Limpo, T. (2015). Progress in written language bursts, pauses, transcription, and written composition across schooling. Scientific Studies of Reading, 19, 374-391. http://dx.doi.org/10.1080/10888438.2015.1059838 
Alves, R. A., Branco, M., Castro, S. L., \& Olive, T. (2012). Effects of handwriting skill, handwriting and dictation modes, and gender of fourth graders on pauses, written language bursts, fluency, and quality. In V. W. Berninger (Ed.), Past, present, and future contributions of cognitive writing research to cognitive psychology (pp. 389-402). New York, NY: Psychology Press.

Alves, R. A., Castro, S. L., \& Olive, T. (2008). Execution and pauses in writing narratives: Processing time, cognitive effort and typing skill. International Journal of Psychology, 43, 969-979. doi:10.1080/00207590701398951

Alves, R. A., Castro, S. L., Sousa, L., \& Strömqvist, S. (2007). Influency of typing skill on pause-execution cycles in written composition. In M. Torrance, L. van Waes, \& D. Galbraith (Eds.), Writing and cognition: Research and applications (pp. 55-65). Amsterdam, the Netherlands: Elsevier.

Alves, R. A., Limpo, T., Fidalgo, R., Carvalhais, L., Pereira, L. A., \& Castro, S. L. (2016). The impact of promoting transcription on early text production: Effects on bursts and pauses, levels of written language, and writing performance. Journal of Educational Psychology, 108, 665-679. http://dx.doi.org/10.1037/edu0000089

Barbier, M. L., \& Spinelli-Jullien, N. (2009). On-line tools for investigating writing strategies in L2. German as a foreign language, 2(3), 23-40.

Chenoweth, N. A., \& Hayes, J. R. (2001). Fluency in writing: Generating text in L1 and L2. Written Communication, 18, 80-98. doi:10.1177/0741088301018001004

Connelly, V., Dockrell, J. E., Walter, K., \& Critten, S. (2012). Predicting the quality of composition and written language bursts from oral language, spelling, and handwriting skills in children with and without Specific Language Impairment. Written Communication, 29, 278-302. doi:10.1177/0741088312451109

Cromley, J. G., \& Azevedo, R. (2006). Self-report of reading comprehension strategies. What are we measuring? Metacognition and Learning, 1, 229-247. http://dx.doi.org/10.1007/s11409-006$\underline{9002-5}$

Foulin, J. N. (1998). To what extent does pause location predict pause duration in adults' and children's writing ? Cahiers de Psychology Cognitive/Current Psychology of Cognition, 17, 601-620.

Hayes, J. R., \& Flower, L. (1980). Identifying the organization of writing processes. In L. W. Gregg \& E. R. Steinberg (Eds.), Cognitive processes in writing (pp. 3-29). Hillsdale, NJ: Erlbaum.

Kaufer, D. S., Hayes, J. R., \& Flower, L. (1986). Composing written sentences. Research in the Teaching of English, 20, 121-140.

Levy, C. M. \& Olive, T. (2002). Real time studies in writing research: Progress and Prospects. En T. Olive \& C. M. Levy (Eds.), Contemporary tools and techniques for studying writing (pp. 1-8). Dordrecht, A.A.: Kluwer Academic Publishers.

Limpo, T., \& Alves, R. A. (2017). Tailoring multicomponent writing interventions: Effects of coupling self-regulation and transcription training. Journal of Learning Disabilities. https://doi.org/10.1177/0022219417708170.

Medimorec, S., \& Risko, E. F. (2017). Pauses in written composition: On the importance of where we pause. Reading and Writing, 30, 1267-1285. doi:10.1007/s11145-017-9723-7

Olive, T. (2010). Methods, techniques, and tools for the on-line study of the writing process. In N. L. Mertens (Ed.), Writing: processes, tools and techniques (pp. 1-18). New York, NY: Nova Science Publishers.

Olive, T., \& Kellogg, R. T. (2002). Concurrent activation of high- and low-level production processes in written composition. Memory \& Cognition, 30, 594-600. doi:10.3758/BF03194960

Olive, T., Alves, R. A., \& Castro, S. L. (2009). Cognitive processes in writing during pause and exe- 


\section{ANÁLISIS DE PAUSAS Y EJECUCIONES PARA EL ESTUDIO DE PROCESOS DE ESCRITURA}

cution periods. European Journal of Cognitive Psychology, 21, 758-785. doi:10.1080/09541440802079850

Prunty, M. M., Barnett, A. L., Wilmut, K., \& Plumb, M. S. (2013). Handwriting speed in children with Developmental Coordination Disorder: Are they really slower? Research in Developmental Disabilities, 34, 2927-2936. doi:10.1016/j.ridd.2013.06.005

Prunty, M. M., Barnett, A. L., Wilmut, K., \& Plumb, M. S. (2014). Research in developmental disabilities: An examination of writing pauses in the handwriting of children with developmental coordination disorder. Research in Developmental Disabilities, 35(11), 2894-2905. doi: 10.1016/j.ridd.2014.07.033.

Schilperoord, J. (2002). On the cognitive status of pauses in discourse production. In T. Olive \& C. M. Levy (Eds.), Contemporary tools and techniques for studying writing (pp. 61-87). Dordrecht, The Netherlands: Kluwer Academic Publishers.

Spelman Miller, K. (2000). Academic writers on-line: Investigating pausing in the production of text. Language Teaching Research, 4, 123-148. http://dx.doi.org/10.1191/136216800675510135

Tillema, M., Van den Bergh, H., Rijlaarsdam, G., \& Sanders, T. (2011). Relating self-reports of writing behavior and online task execution using a temporal model. Metacognition Learning, 6, 229253. doi: 10.1007/s11409-011-9072-x.

Torrance, M., Rønneberg, V., Johansson, C., \& Uppstad, P. H. (2016). Adolescent weak decoders writing in a shallow orthography: Process and product. Scientific Studies of Reading, 20(5), 375-388. doi: 10.1080/10888438.2016.1205071

Van der Pool, E. (1996). A text-analytical study of conceptual writing proceses and their development. En G. Rijlaarsdam, H. van der Bergh \& M. Couzijn (Eds.), Theories, Models and Methodology in Writing Research (pp. 456-472). Amsterdam: Amsterdam University Press.

Van Hell, J., Verhoeven, L. \& van Beijsterveldt, L. (2008). Pause time patterns in writing narrative and expository texts by children and adults. Discourse Processes, 45, 406-427. http://dx.doi.org/10.1080/01638530802070080

Van Waes, L., \& Schellens, P. J. (2003). Writing profiles: The effect of the writing mode on revision patterns of experienced writers. Journal of Pragmatics, 35, 829-853. http://dx.doi.org/10.1016/S0378-2166(02)00121-2

Van Waes, L., Leijten, M., Wengelin, A., \& Lindgren, E. (2012). Logging tools to study digital writing processes. In V. W. Berninger (Ed.), Past, present, and future contributions of cognitive writing research to cognitive psychology (pp. 507-533). New York, NY: Psychology Press.

Wengelin, A. (2006). Examining pauses in writing: Theory, methods and empirical data. In K. Sullivan \& E. Lindgren (Eds.), Computer keystroke logging and writing: Methods and applications (pp. 107-130). Amsterdam, Netherlands: Elsevier.

Wengelin, A. (2007). The word-level focus in text production by adults with reading and writing difficulties. In M. W. Torrance, L. van Waes, \& D. Galbraith (Eds.), Writing and cognition: Research and applications (Vol. 20, pp. 67-82). Oxford, United Kingdom: Elsevier. 